\title{
DINÁMICA ESPACIO TEMPORAL DE OCURRENCIA DE INCENDIOS EN ZONAS CON DIFERENTES TIPOS DE MANEJO EN EL NOROESTE DE LA AMAZONIA: ¿BARRERA EFECTIVA?
}

\section{TEMPORAL-SPACE DYNAMICS OF FIRE OCCURRENCE IN ZONES WITH DIFFERENT TYPES OF MANAGEMENT IN NORTHWEST AMAZON: EFFECTIVE BARRIER?}

\author{
J. Sebastian BARRETO,*, Tania M. GONZÁLEZ', Dolors ARMENTERAS ${ }^{1}$ \\ ${ }^{1}$ Grupo de Ecología del Paisaje y Modelación de Ecosistemas, Departamento de Biología, \\ Facultad de Ciencias, Universidad Nacional de Colombia, Sede Bogotá, Edificio 421, Oficina \\ 223. Cra 30 n. ${ }^{\circ} 45-03$, Bogotá, Colombia \\ *Autor Corresponsal. E-mail: jsbarretor@unal.edu.co
}

Historia del Artículo

Recibido: Julio 19, 2016 Evaluado: Diciembre 29, 2016 Aceptado: Enero 19, 2017 Disponible: Febrero 07, 2017

Resumen |

La acción del hombre y la variabilidad climática asociada al calentamiento global está afectando la incidencia de fuegos en las regiones tropicales, aumentando tanto su intensidad como su frecuencia, particularmente en periodos de sequías. En este estudio se realizó un análisis espacio temporal para identificar la ocurrencia de fuegos dentro y fuera de áreas protegidas en el noroeste de la Amazonía. El objetivo fue analizar la efectividad de estas figuras de protección o manejo en los países de la región en términos de reducción de la afectación por incendios. Se utilizaron datos de focos activos de fuegos detectados por sensores remotos (MODIS) para el periodo comprendido entre diciembre del año 2000 hasta febrero del año 2015 en Brasil, Colombia, Ecuador, Perú y Venezuela. Se siguió la denominación asignada por La Unión Internacional para la Conservación de la Naturaleza (IUCN), las áreas protegidas presentes en la región se agruparon en tres categorías, y la dinámica de los incendios fue analizada a diferentes niveles de estudio. Los resultados indican que las áreas protegidas presentan en todos los casos una menor densidad de fuegos, a diferencia de territorios sin ningún tipo de protección. En términos de comparación de la efectividad, se presenta una mayor cantidad de fuegos en la categoría de resguardo indígena, mientras que la mayor densidad de fuegos por $\mathrm{km}^{2}$ ocurre en áreas tipo categorías II-III. Los resultados obtenidos mostraron que la presencia de áreas protegidas en el noroeste de la Amazonía es efectiva para mitigar la incidencia de fuegos forestales.

Palabras Clave: Focos activos, incendios de vegetación, parques naturales, IUCN, efectividad.

Abstract

Human activity and climate variability associated with global warming is affecting the incidence of fires in tropical regions, increasing both their intensity and frequency, particularly in periods of drought. In this study a spatiotemporal analysis was performed to identify the occurrence of fires inside and outside protected areas in the northwest of the Amazon. The objective was to analyze the effectiveness of these forms of protection or management in the countries of the region in terms of reduced fire occurrence. We use active fires occurrences detected by remote sensing (MODIS) for the period from December 2000 to February 2015 in Brazil, Colombia, Ecuador, Peru and Venezuela. We based our study in the typology of protected areas assigned by the International Union for Conservation of Nature (IUCN), those were grouped into three categories and the fire dynamics was analyzed at different levels of study. The results indicate that protected areas present in all cases a lower density of fires in comparison with territories without any protection. In terms of comparison of the effectiveness, a greater number of fires is presented in the category of indigenous reservation, while the highest density of fires per km2 occurring in areas of category II -III. The results showed that the presence of protected areas in the northwest of the Amazon is effective to mitigate the incidence of forest fires.

Keywords: fire hotspots, vegetation fires, national parks, IUCN, effectiveness 


\section{INTRODUCCIÓN |}

El Neotrópico es quizás la zona más diversa del planeta, en donde se encuentran varios puntos calientes de biodiversidad, que son de importancia a nivel mundial por la riqueza de especies, el endemismo y las amenazas que enfrentan (Ceballos y Ortega-Baes, 2011). Adicionalmente los ecosistemas presentes en el Neotrópico desempeñan un papel importante para muchos procesos ecológicos y para el bienestar humano (Oliveira et al. 2007) a través de la prestación de múltiples servicios ecosistémicos (Ceballos y Ortega-Baes, 2011). En este sentido, la región amazónica representa un depósito no solo de biodiversidad, sino también de carbono, jugando un importante papel en la regulación climática de la zona (Chomitz y Thomas, 2003). No obstante, es una región que exhibe grandes amenazas siendo sujeta a altas tasas de deforestación debido a la conversión de tierras para la agricultura, los asentamientos humanos y la extracción de madera (Armenteras et al. 2006; Oliveira et al. 2007), además de verse afectada por el calentamiento global (Fearnside, 2009).

Una amenazadora sinergia entre la degradación de los bosques causada por prácticas de explotación, la fragmentación del bosque y las sequías cada vez más severas han vuelto muchos bosques tropicales amazónicos y del sudeste de Asia más propensos a los incendios (FAO, 2013). Estos procesos conllevan a la degradación del ambiente, la destrucción del paisaje, erosión del suelo, agotamiento de nutrientes, perdida de la biodiversidad, cambios ecosistémicos y contaminación del aire (Pereira et al. 2012). En el Amazonas, como en otras zonas de los trópicos, las actividades humanas han alterado la dinámica de los incendios. Los bosques presentes en la región están bajo un constante régimen de fuegos, producidos naturalmente por las sequías y altas temperaturas, así como por acción antrópica. El régimen del fuego está fuertemente influenciado por valores culturales, practicas humanas, uso del fuego, control de fuegos y políticas regionales y nacionales (White et al. 2011). Todos estos causantes producen una alteración en la intensidad, tamaño, frecuencia y duración de este disturbio, y si se llega a exceder la resiliencia o recuperación del ecosistema en que se presenta, la alteración del mismo puede ser dramática (Holling, 1973). El uso del fuego para la limpieza y el manejo del suelo es una de las mayores amenazas para los bosques neotropicales y una de las fuentes más importantes de emisiones carbono a la atmósfera (Cochrane y Laurance, 2008; Morton et al. 2008; Aragão et al. 2008).

Dentro de los mecanismos implementados por el hombre para afrontar los cambios ecológicos y la pérdida de biodiversidad, se ha reconocido la importancia que presentan las áreas protegidas (Tittensor et al. 2014). Estas áreas funcionan como medios de protección y aislamiento ante el avance de los procesos de colonización y urbanización. Las áreas protegidas no solo proveen hábitat para especies amenazadas, también contribuyen al sostenimiento de comunidades locales, a generar ingresos a través del ecoturismo, lo que impulsa economías locales y nacionales, y de igual manera, desempeñan un papel clave en los procesos de mitigación y adaptación al cambio climático (Ervin et al. 2010). Hasta un $70 \%$ de las personas más pobres del mundo dependen de manera crítica de la biodiversidad, la cual proporciona alimentación, agua, refugio, medicinas y un medio de sustentabilidad económica (Secretariat of the Convention on Biological Diversity, 2009). Gracias al esfuerzo de agencias gubernamentales y no gubernamentales durante las últimas décadas, las áreas terrestres protegidas en el mundo abarcaban más de 18 millones de $\mathrm{km}^{2}$ para el 2010, en comparación con poco más de los dos millones de $\mathrm{km}^{2}$ en 1970 (WCMC, 2010).

Se ha evidenciado que la transformación del bosque es menos intensiva dentro de áreas protegidas, no solo dentro del límite de estas, sino también, en las zonas circundantes a estas figuras de protección (Armenteras et al. 2009b). Particularmente, en el caso de la Amazonía brasilera, el efecto inhibitorio de tierras indígenas sobre la deforestación es enérgico, y estas representan la barrera más importante ante este proceso (Nepstad et al. 2006). En cuanto a las diferentes categorías de áreas protegidas, aquellas designadas como I y II, parecen ser más efectivas reduciendo la deforestación, en comparación con áreas tipo V-VI que incluyen un enfoque de manejo sostenible (Campbell et al. 2008). Para el caso de la incidencia de fuegos, algunos estudios han mostrado que estos ocurren con menor frecuencia en áreas protegidas o reservas con un bajo impacto humano (Adeney et al. 2009; Armenteras et al. 2013).

A pesar que hay avances considerables en Brasil y parciales en Colombia, fuera de estos dos, en los países del noroeste de la Amazonia no hay estudios que muestren el grado de efectividad de las áreas protegidas para mitigar incendios forestales. El noroeste de la Amazonía cuenta con varias áreas protegidas o de manejo especial distribuidas en cinco países, convirtiéndose en un medio útil para la conservación de esta región, además de servir como un indicador de la eficacia de la protección (Adeney et al. 2009). El objetivo de este estudio es realizar un análisis espacio temporal de los incendios forestales y analizar la efectividad para reducir su ocurrencia por parte de las diferentes áreas protegidas presentes en el noroeste de la Amazonía. Con el fin de evaluar esta efectividad, se comparó la eficacia de zonas protegidas relacionadas con la población indígena (resguardos, tierras y reservas indígenas), las áreas con proyecciones de protección de ecosistemas (categoría II IUCN) y protección de rasgos naturales (categoría III IUCN), y áreas que promueven el uso sostenible de recursos naturales (categoría VI IUCN) (Dudley y Solton, 2008). Para responder sobre la eficacia se evaluó si estas áreas de gestión y de conservación en el noroeste de la Amazonia se comportan de forma similar en toda la región, si hay diferencias en el 
norte y sur del ecuador y, finalmente entre todos los países que hacen parte del área de estudio.

\section{MATERIALES Y MÉTODOS}

\section{Área de estudio}

El área de estudio (Fig. 1) corresponde al noroeste de la Amazonía $\left(65^{\circ} \mathrm{W}, 10^{\circ} \mathrm{S}-6^{\circ} \mathrm{N}\right)$, que comprende regiones de los países de Brasil, Colombia, Ecuador, Perú y Venezuela. El área total es de aproximadamente $2.164 .132 \mathrm{~km}^{2}$, de los cuales $574.287 \mathrm{~km}^{2}$ se encuentran ubicados al norte del Ecuador, $\mathrm{y}$ 1'590.179 al sur de este. Los países con mayor área son Brasil (42\%) con $897.178 \mathrm{~km}^{2}$, seguido de Perú $\left(26 \%, 569.759 \mathrm{~km}^{2}\right)$ y Colombia $\left(21 \%, 454.050 \mathrm{~km}^{2}\right)$ y por último Venezuela $\mathrm{y}$ Ecuador con $\left(8 \%, 168.751 \mathrm{~km}^{2}\right)$ y $\left(3 \%, 168.751 \mathrm{~km}^{2}\right)$ respectivamente.
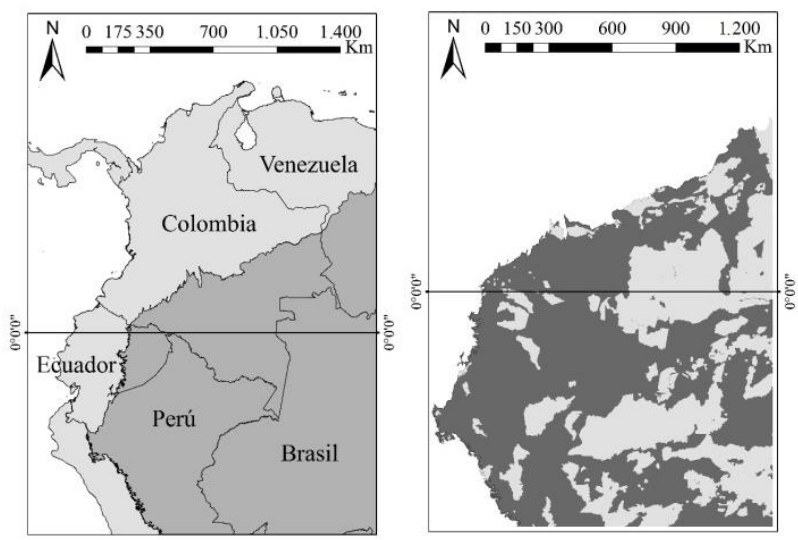

Figura 1. Área de estudio a la izquierda en color gris oscuro (noroeste de la Amazonía). A la derecha zona de estudio con áreas protegidas en color gris claro.

\section{Fuente de datos}

Los datos de fuegos son focos activos o anomalías térmicas detectadas por el sensor MODIS, descargados a través de FIRMS (Fire Information of Resource Management System Active Fire Data, Collection 5.1) para el tiempo comprendido entre noviembre del 2000 hasta febrero del 2015. La detección del foco activo de fuego se realiza a través de un algoritmo contextual que utiliza la fuerte emisión de radiación en el infrarrojo medio de los incendios (Giglio et al. 2003).

La información de áreas protegidas para el área de estudio se obtuvo a partir de La Base de Datos Mundial de Áreas Protegidas (WDPA, 2015). Dentro del noroeste de la Amazonía se encuentran 341 áreas protegidas, para este estudio se tomaron aquellas áreas que bien, pertenecen a alguno de los tres siguientes grupos (Tabla 1): 1) Resguardos indígenas: dentro de esta categoría se incluyen áreas tipo resguardo indígena, reserva indígena y tierra indígena. 2) Categorías II y III IUCN, ambas categorías se agruparon en una sola, debido a la similitud en políticas de manejo de ambas (Nelson y Chomitz, 2011). 3) Categoría VI IUCN.
Las áreas protegidas categoría II hace referencia a Parques Nacionales, los cuales están destinados a la protección de ecosistemas, o a la protección del patrimonio cultural, son grandes áreas naturales que tienen como objetivo proteger procesos ecológicos a gran escala, junto con las especies y ecosistemas característicos del área, los cuales ofrecen oportunidades científicas, culturales, espirituales y recreacionales (Borrini-Feyerabend et al. 2013). Por otro lado, las áreas categoría III hacen referencia a Monumentos Naturales, estas zonas son establecidas para la protección de un monumento natural especifico, generalmente son de pequeña extensión, pero con un alto valor histórico o cultural (Borrini-Feyerabend et al. 2013).

Las áreas categoría VI son áreas protegidas para el manejo de recursos, las cuales son destinadas principalmente para el uso sostenible de ecosistemas naturales (Dudley, 2008). La categoría VI de áreas protegidas es percibida como una categoría de manejo que reconoce la necesidad de conservar la biodiversidad, y al mismo tiempo, hacer posible el aprovechamiento sostenible de los recursos naturales existentes por parte de la población local (Ponce y Curonisy, 2008a).

Las áreas indígenas son áreas naturales y/o ecosistemas modificados que contienen valores significativos de biodiversidad, valores ecológicos y valores culturales, conservados de manera voluntaria por indígenas, nómadas o comunidades locales a través de normas y costumbres tradicionales $u$ otras formas efectivas de manejo (BorriniFeyerabend et al. 2004). Es vital considerar que las poblaciones locales tienen fuertes lazos con la tierra, tal es el caso de los pueblos indígenas, quienes aparecen como los mejores aliados en programas de conservación debido a su permanente presencia en la región, además los cambios que estos causan en su entorno son mucho más pequeños que los impactos ambientales generados por otros factores sociales (Fernández-Baca y Martin, 2007).

\begin{tabular}{|c|c|c|c|}
\hline & $\begin{array}{c}\text { Resguardo } \\
\text { indígena }\end{array}$ & $\begin{array}{c}\text { Categorías II y III } \\
\text { IUCN }\end{array}$ & Categoría VI IUCN \\
\hline Brasil & 134 & 7 & 25 \\
\hline Colombia & 66 & 8 & 3 \\
\hline Ecuador & 2 & 4 & 3 \\
\hline Perú & 10 & 9 & 13 \\
\hline Venezuela & 2 & 7 & 4 \\
\hline
\end{tabular}

\section{Análisis de la información}

Se empleó el programa ArcMap 10.2 de ArcGIS ${ }^{\circledR}$ con el cual se identificaron los fuegos que ocurrían dentro o fuera de las tres categorías de áreas protegidas (Figura 1). Posterior a esto, con los fuegos al interior de las áreas, se identificó cuales se presentaban dentro de áreas tipo resguardo indígena, o si se presentaban en áreas pertenecientes a la categoría II- III IUCN, o a la categoría IV IUCN, con el fin de analizar la efectividad de las áreas 
protegidas en la mitigación de la ocurrencia de incendios forestales.

Los análisis se realizaron en tres niveles, de acuerdo a la pertinencia de los datos: i) dentro de cada uno de los países que componen la región del noroeste de la Amazonía ii) en las porciones sur/norte del área de estudio, y iii) entre países.

\section{RESULTADOS Y DISCUSIÓN |}

Para el noroeste de la Amazonía, aproximadamente 91,4 $\%$ de los fuegos detectados entre el año 2000-2015 (244.633 focos) se presentaron fuera de alguna de las tres categorías de protección, mientras que el restante $8,6 \%$ de los fuegos (22.932 focos) ocurrieron dentro de estas áreas. Para los fuegos que coincidieron en áreas con alguna de las tres categorizaciones empleadas en este estudio, aproximadamente $42,0 \%$ de estos fuegos ocurrieron dentro de áreas clasificadas como resguardos indígenas, 36,0\% y $22,0 \%$ dentro de áreas clasificadas según la IUCN en la categoría VI, y II-III respetivamente. En relación a la densidad de fuegos, esta fue bastante similar entre las clasificaciones II-III IUCN y VI IUCN. Ocurrieron 3,83 incendios por cada 100 $\mathrm{km}^{2}$ en las áreas protegidas clasificadas como categorías II-III IUCN, y 3,82 fuegos por cada $100 \mathrm{~km}^{2}$ en áreas categoría VI IUCN, por último, se presentaron 2,21 eventos por cada 100 $\mathrm{km}^{2}$ en áreas categoría resguardo indígena.

La densidad de fuegos fuera de áreas protegidas fue de aproximadamente seis veces, superior a los promedios dentro de las áreas de manejo estudiadas, con 17,93 fuegos por $100 \mathrm{~km}^{2}$, en comparación con la densidad dentro de las áreas, que correspondió a 2,87 incendios por cada 100 km².

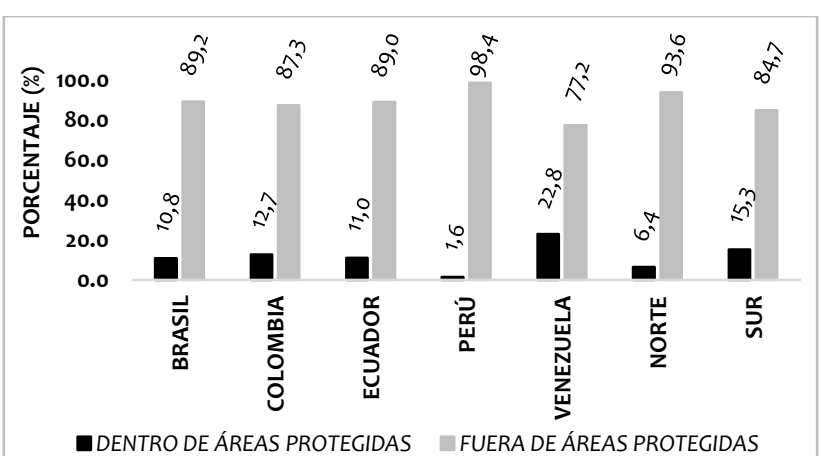

Figura 2. Porcentaje de ocurrencia de fuegos dentro y fuera de áreas protegidas por país, en el hemisferio norte y sur de la zona de estudio, para todo el período de tiempo.

Al analizar el comportamiento por país, y al sur-norte del área de estudio, los resultados mostraron que se mantuvo la relación de fuegos dentro y fuera de áreas protegidas, siempre fue mayor la incidencia de fuegos fuera de estas áreas en estos dos análisis contrastantes (Fig. 2). Se presentó un mayor porcentaje de fuegos dentro de áreas protegidas en Venezuela (22,8\%), seguido de Colombia, Ecuador y Brasil (con $12,7 \%, 11,0 \%$ y $10,8 \%$ respectivamente), y finalmente, en el país donde se presentó el menor porcentaje de fuegos en áreas protegidas fue Perú, con 1,6\%. En cuanto a las zonas al norte y sur del Ecuador, más del doble (15,3\%) de fuegos se presentaron dentro de áreas protegidas en la parte norte del área de estudio, a diferencia de la parte el sur $(6,4 \%)$.

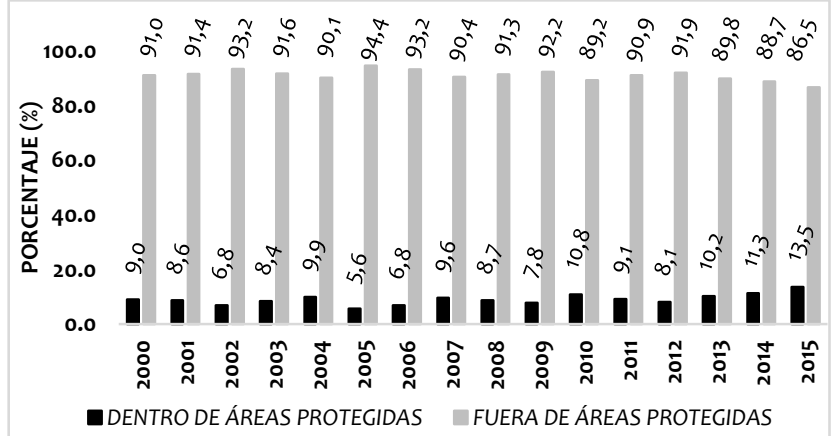

Figura 3. Incidencia de fuegos dentro y fuera de áreas protegidas por cada año

Al analizar la dinámica temporal de los fuegos detectados por MODIS, se observa que, para todos los años, la proporción de fuegos que ocurrieron dentro de áreas protegidas siempre fue menor, en comparación con la proporción fuera de estas áreas, pero varía entre años. El año donde se presentó un menor número de fuegos dentro de áreas fue 2005 (5,6\%), mientras que el año donde se presentó la mayor cantidad de fuegos dentro de las áreas fue $2015(13,5 \%)$.

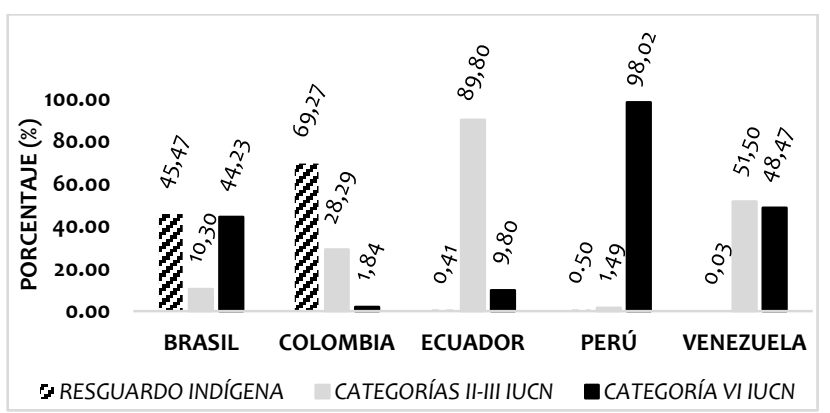

Figura 4. Fuegos dentro de áreas protegidas en cada país de la zona de estudio, para todo el período de tiempo.

Sobre la ocurrencia de incendios por tipo de área de manejo, en Brasil se observó una proporción entre los fuegos que ocurrieron en resguardos indígenas y en áreas tipo categoría VI IUCN (45,47\% y 44,23\% respectivamente), mientras que solo el 10,30\% de fuegos ocurrieron en áreas categoría II-III (Fig. 4). Para Colombia, la mayoría de fuegos ocurrieron en aquellas áreas clasificadas como resguardo indígena $(69,27 \%)$, seguido de fuegos en áreas categoría II-III $(28,89 \%)$, y, por último, un menor porcentaje de incendios $(1,84 \%)$ en áreas categoría VI. En Ecuador la mayoría de los incendios se presentaron en la categoría II- III (89,8\%), 9,80\% en áreas de resguardo indígena y $0,41 \%$ en áreas categoría VI. En Perú la gran mayoría de fuegos $(98,02 \%)$ sucedieron en áreas categoría VI, 1,49\% en categoría II-III, y tan solo el 0,41\% de los fuegos en áreas tipo resguardo indígena. En cuanto a Venezuela, la mayoría de fuegos ocurrieron dentro de áreas 
categoría II-III (51,50\%), seguido de áreas categoría VI $(48,47 \%)$ y $0,03 \%$ en resguardos indígenas. En la tabla 2 se encuentra el resumen de resultados de densidad para cada categoría de áreas protegidas en cada país de la zona de estudio.

En términos de distribución espacial, las regiones con una mayor densidad de fuegos por $\mathrm{km}^{2}$ se presentaron fuera de áreas protegidas (Fig. 5).

Los resultados de este estudio muestran la efectividad relativa de las áreas de manejo estudiadas para mitigar la ocurrencia de fuegos en el noroeste de la Amazonía, aunque la ocurrencia de incendios no es nula, no se logra una efectividad completa. Este patrón se ha mantenido a lo largo del periodo analizado. En otros estudios realizados en Brasil han reportado un comportamiento similar, la densidad de fuegos fuera de áreas protegidas es mayor que la que se presenta dentro del perímetro de parques naturales y tierras indígenas (Nepstad et al. 2006). En el caso del noroeste de la Amazonía, los bosques presentes en áreas protegidas y reservas indígenas se encuentran menos fragmentados, y es posible que esta característica, junto con la dificultosa accesibilidad, los haga menos susceptibles al fuego (Armenteras et al. 2013). En todos los países, los resultados confirman que las áreas bajo alguna figura de manejo especial sirven como barrera contra incendios (Armenteras et al. 2009b), por lo tanto, la presencia de áreas protegidas puede considerarse como un mecanismo valido para mitigar la incidencia de fuegos forestales. Las áreas protegidas en la región Amazónica, contribuyen a reducir en un 10\% el riesgo de afectación del cambio climático sobre el bioma de la Amazonía (Suarez et al. 2015). Por este motivo es altamente importante fortalecer este tipo de estrategias.

Dentro de los incendios que efectivamente ocurrieron en el territorio bajo alguna figura de protección o manejo, se presentó un mayor número de fuegos en las áreas tipo resguardo indígena. Hay que tener en cuenta el gran número de áreas de este tipo presentes en el noroeste de la Amazonía (194). Esta mayor ocurrencia se explica por las prácticas agrícolas y de subsistencia de las comunidades indígenas que habitan este tipo de áreas protegidas, donde se emplea frecuentemente el mecanismo de tala y quema para la limpieza y adecuación de la tierra y preparación de pequeñas chagras o cultivos (Armenteras et al. 2009a). Sin embargo, es importante considerar que, a pesar del gran número de eventos de fuego y extensión de estas áreas, es en estas donde se presenta el menor número de fuegos por $100 \mathrm{~km}^{2}(2,21)$.

La densidad de fuegos fue bastante similar para áreas categoría II-III y áreas categoría VI (3,83 y 3,82 fuegos por cada $100 \mathrm{~km}^{2}$, respectivamente). Sin embargo, las áreas categoría III que representan una extensión significativamente menor, presentaron una gran densidad de fuegos. Es importante considerar que tanto las áreas categoría II, como las áreas categoría III normalmente incluyen (o prevén) actividades turísticas y/o recreativas, lo que incluye el desarrollo de la infraestructura necesaria para este fin (Dudley, 2008). En áreas categoría VI se ejerce una protección no estricta, donde el objetivo principal es la protección de ecosistemas y uso de recursos naturales de manera sostenible, junto con una estrategia de gestión múltiple del suelo (Nelson y Chomitz, 2011). La categoría VI de la UICN en América Latina es muy variada, sin embargo, es evidente que dentro de esta variedad la mayoría de países cuentan con las categorías de Reserva Forestal (bajo distintas nomenclaturas) destinadas básicamente a la producción de madera o productos forestales en general (Ponce y Curonisy, 2008a). En general, para América Latina, los problemas que presentan las áreas protegidas categoría VI, están relacionados con conflictos de tenencia de tierras, la falta de recursos financieros, limitada capacidad de gestión del estado y los proyectos de alto impacto sobre el medio ambiente, entre otros (Ponce y Curonisy, 2008b).

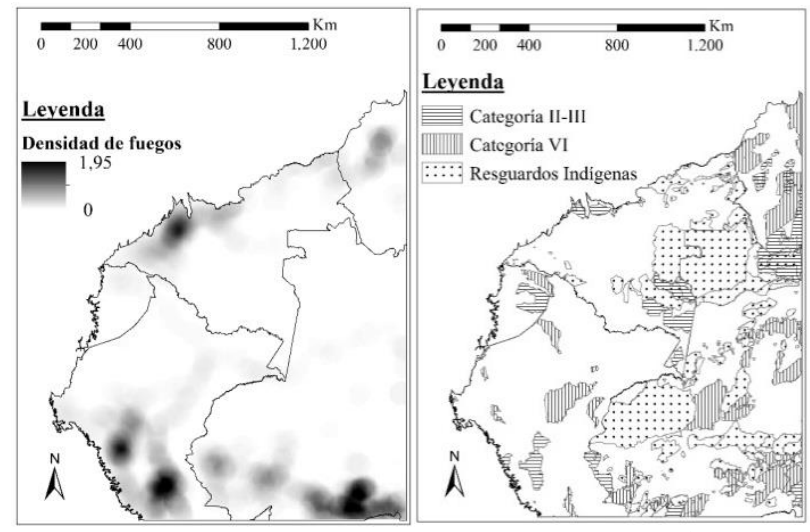

Figura 5. Densidad de fuegos (número de fuegos por $\mathrm{km}^{2}$ ) y áreas protegidas en el noroeste de la Amazonía.

Tabla 2. Resumen de resultados para cada país del noroeste de la Amazonía. NA: número de áreas o fragmentos de estas, AT: área total (en km²), NF: número de fuegos, D: densidad de fuegos (números de fuegos por cada $100 \mathrm{~km}^{2}$ )

\begin{tabular}{|c|c|c|c|c|c|c|c|c|c|c|c|c|}
\hline & \multicolumn{4}{|c|}{ RESGUARDO INDÍGENA } & \multicolumn{4}{|c|}{ CATEGORÍAS II-III IUCN } & \multicolumn{4}{|c|}{ CATEGORÍAVI IUCN } \\
\hline & NA & AT & NF & D & NA & AT & NF & D & NA & AT & $\mathrm{NF}$ & D \\
\hline BRASIL & 134 & $344.874,51$ & 5.321 & 1,54 & 7 & $40.831,18$ & 1.205 & 2.95 & 25 & $118.638,71$ & 5.176 & 4,36 \\
\hline COLOMBIA & 66 & $100.630,77$ & 4.511 & 4,48 & 8 & $35.443,78$ & 1.881 & 5.31 & 3 & 486,29 & 120 & 24,68 \\
\hline ECUADOR & 2 & 10,32 & 1 & 9,69 & 4 & $10.590,19$ & 220 & 2.08 & 3 & $5 \cdot 932,63$ & 24 & 0,40 \\
\hline PERU & 10 & 151,51 & 7 & 4,62 & 9 & $17.185,24$ & 21 & 0.12 & 13 & $39.537,75$ & 1.383 & 3,50 \\
\hline VENEZUELA & 2 & 127,58 & 1 & 0,78 & 7 & $30.161,68$ & 1.819 & 6.03 & 4 & $55.802,28$ & 1.712 & 3,07 \\
\hline
\end{tabular}


En términos de los países, el mayor número de fuegos por cada $100 \mathrm{~km}^{2}(24,68)$ se presentó para áreas tipo categoría VI IUCN en Colombia, que corresponden a dos áreas designadas como Reservas Forestales Protectoras. Estas dos áreas son la "Serranía del Capricho, Mirolindo y Cerritos", y la otra "Cuenca de los caños La Esperanza, Agua Bonita, Negro, La María y La Lindosa" ambas ubicadas en el departamento del Guaviare, con $408,9 \mathrm{~km}^{2}$ y $71,8 \mathrm{~km}^{2}$ respectivamente. Desde el plan básico de ordenamiento territorial para el municipio de San José del Guaviare en el año 2000 , se reconocen problemas relacionados con la ocupación y uso inadecuado de las tierras para el sector de Mirolindo, El capricho y La Lindosa (POTB Municipio de San José del Guaviare, 2000). Las áreas protegidas más afectadas son la Zona de Preservación de La Lindosa, los cerros El Capricho, la reserva de caños como La María, La Esperanza, Agua bonita y Caño Negro, estas presentan una fuerte deforestación debido al alto grado de intervención antrópica (Plan de desarrollo del Guaviare, 2004). Para la Amazonía Colombiana, en el año 2012, en el departamento del Guaviare, se presentó la mayor área en bosques fragmentados (Murcia et al. 2014), factor que aumenta la susceptibilidad del bosque ante eventos de fuego.

La menor densidad de fuegos (0,12 fuegos por cada 100 $\mathrm{km}^{2}$ ) se presentó en áreas categorías II-III IUCN en Perú. Para esta categoría, se presentaron cinco áreas, cuatro con designación de Parque Nacional y una Santuario Natural. Estas áreas son: Río Abiseo $\left(1.943,8 \mathrm{~km}^{2}\right)$, Ichigkat Muja Cordillera del Cóndor $\left(861,3 \mathrm{~km}^{2}\right)$, Tingo María $\left(49,4 \mathrm{~km}^{2}\right)$, Cordillera Azul $\left(13879,1 \mathrm{~km}^{2}\right)$ y Tabaconas Namballe $(312,7$ km2). El parque Nacional Río Abiseo es un área inscrita como Sitio de Patrimonio Mixto, respecto a este tipo de sitios, el Reglamento establece que son áreas estrictamente delimitadas, reconocidas internacionalmente en la Lista del Patrimonio Mundial administrada por el Comité del Patrimonio Mundial de la UNESCO (Solano, 2009). En el caso de Cordillera Azul, destaca el inventario biológico del lugar, en una experiencia de trabajo de tan solo tres semanas, se encontraron más de 21 especies nuevas para la ciencia (Alverson et al. 2001). La gestión exitosa en el Parque Nacional Cordillera Azul se debe en gran parte a la gestión financiera centralizada a través de CIMA (Centro de Conservación, Investigación y Manejo de Áreas Naturales), que ha permitido canalizar la disponibilidad de fuentes de financiamiento externa, que han apoyado con fondos significativos a la gestión del área protegida desde su creación, dentro de la cual destaca el proyecto REDD+ como instrumento de financiación (Araujo y Casavecchia, 2014).

\section{CONCLUSIONES |}

En este trabajo se presenta una aproximación a la ocurrencia de incendios en diferentes áreas de manejo presentes en el noroeste de la Amazonía. Los resultados mostraron que hay una efectividad parcial de las áreas protegidas como mecanismo para mitigar la incidencia de incendios forestales en la región. Si bien la incidencia de fuegos se reduce, no es evitado por completo este tipo de disturbio. Por lo tanto, aunque la creación de un área protegida nace a partir de la necesidad de preservación, estas aportan un servicio adicional a los ecosistemas naturales, mitigando la incidencia de fuegos y las consecuentes perdidas ecológicas y económicas que derivan de estos. Es evidente que independientemente del tipo de área protegida la presencia de fuegos dentro de estas es menor, en comparación con los eventos de fuego que se presentan fuera de estas áreas, lo cual se puede observar en todos los contrastes de comparación empleados en este estudio. Igualmente, la incidencia de este tipo de eventos varía dependiendo del tipo de figura de manejo, pues son diferentes los medios generales de gestión administrativa en torno al objetivo de conservación, dentro de lo cual es importante considerar la administración local de cada una de las entidades, como por ejemplo el Parque Nacional Cordillera Azul, en Perú, donde la eficiencia administrativa ha logrado la captación de ingresos gracias a la implementación de proyectos de investigación que sustentan económicamente la gestión de este parque, lo que puede ser empleado como un modelo para otros escenarios. Esto en comparación con áreas donde se reconocen problemáticas ambientales y sociales que si bien, tienen una relación directa con la incidencia de fuegos, no logran captar la atención necesaria para subsanarlas. No obstante, y dado que no se logra una efectividad total, es importante incorporar las dinámicas temporales (asociadas a variabilidad climática) de los incendios en esfuerzos futuros de conservación que se planteen para la región. Entre estos se recomienda la creación de sistemas locales de monitoreo de incendios, alertas tempranas y planes de control de incendios forestales, que contribuyan en el control de la generación de incendios ocasionados por el hombre durante los periodos secos, además de planificar acciones para mitigar los impactos generados por este disturbio, enfatizando e implementando políticas socio-económicas que garanticen la sostenibilidad económica en las zonas alrededor de estas áreas, debido a que es indispensable contar con los recursos suficientes para realizar una gestión eficaz.

\section{AGRADECIMIENTOS}

Los autores desean agradecer al Programa Nacional de Iniciación en Investigación, Creación e Innovación de la Universidad Nacional de Colombia 2013-2015 (Código 29122), por el apoyo al proceso de investigación, igualmente, al sistema FIRMS y a la WDPA, que proveen la información acerca de fuegos y áreas protegidas, respectivamente.

\section{REFERENCIAS}

- Adeney JM, Christensen NL, Pimm SL. 2009. Reserves Protect against Deforestation Fires in the Amazon. Plos One, 4:1-12. 
- Alverson W, Rodríguez L, Moskovits V editores). 2001. Perú: Biabo Cordillera Azul. Rapid Biological Inventories Report 2. The Field Museum, Chicago, 72p.

- Aragão LE, Malhi Y, Barbier N, Lima A, Shimabukuro Y, Anderson L, Saatchi S. 2008. Interactions between rainfall, deforestation and fires during recent years in the Brazilian Amazonia. Philosophical Transactions Of The Royal Society B, 363:1779-1785.

- Araujo N y Casavecchia C. 2014. Parque Nacional Cordillera Azul, estudio de caso Perú. En: Amazonía más allá de las fronteras: lecciones aprendidas en áreas protegidas. UICN, Quito, Ecuador, 18p.

- Armenteras D, Rudas G, Rodriguez N, Sua S, Romero M. 2006. Patterns and causes of deforestation in the Colombian Amazon. Ecological Indicators, 6:353-368.

- Armenteras D, González-Alonso F, Franco C. 2009a. Distribución geográfica y temporal de incendios en Colombia utilizando datos de anomalías térmicas. Caldasia, 31(2):303-318.

- Armenteras D, Rodríguez N, Retana J. 2009b. Are conservation strategies effective in avoiding the deforestation of the Colombian Guyana Shield?. Biological Conservation, 142:1411-1419.

- Armenteras D, González TM, Retana J. 2013. Forest fragmentation and edge influence on fire occurrence and intensity under different management types in Amazon forests. Biological Conservation, 159:73-79.

- Borrini-Feyerabend, G, Kothari A, Oviedo G. 2004. Indigenous and Local Communities and Protected Areas: towards Equity and Enhanced Conservation, volume 11 of the IUCN Best Practice in Protected Area Management Series, Gland, Switzerland and Cambridge, UK, 139p.

- Borrini-Feyerabend, G, Dudley N, Jaeger T, Lassen B, Pathak-Broome N, Phillips A, Sandwith T. 2013. Governance of Protected Areas: From understanding to action. Best Practice Protected Area Guidelines Series No. 20, Gland, Switzerland, IUCN, 124pp.

- Campbell A, Clark S, Coad L, Miles L, Bolt K, Roe D. 2008. Protecting the future: Carbon, forests, protected areas and local livelihoods. Biodiversity, (9):117-121.

- Ceballos G y Ortega-Baes P. 2011. La sexta extinción: la perdida de especies y poblaciones en el Neotrópico. P. 95-108. Capítulo VI. Conservación Biológica: Perspectivas de Latinoamérica. Editorial Universitaria, Chile, 196p.

- Chomitz K y Thomas T. 2003. Determinants of Land Use in Amazônia: A FineScale Spatial Analysis. American Journal of Agricultural Economics, 85(4):1016-1028.

- Cochrane MA y Laurance WF. 2008. Synergisms among fire, land use, and climate change in the Amazon. Ambio, 37(7-8):522-527.

- Dudley N (Editor). 2008. Directrices para la aplicación de las categorías de gestión de áreas protegidas. UICN, Gland, Suiza, 96p.

- Dudley N y Solton S. 2008. Defining protected areas: an international conference in Almeria, Spain.UICN, Gland, Switzerland, 220p.

- Ervin J, Sekhran N, Dinu A, Gidda S, Vergeichik M, Mee J. 2010. Protected Areas for the 21st Century: Lessons from UNDP/GEF's Portfolio. New York: United Nations Development Programme and Montreal: Convention on Biological Diversity; 2010

- FAO. 2013. Estudio FAO Montes N ${ }^{\circ}$ 172. Organización de las Naciones Unidas para la Alimentación y la Agricultura, Roma, Italia, 111p.

- Fearnside, PM. 2009. Global warming in Amazonia: Impacts and Mitigation. Acta Amazónica, 39(4):1003-1012.

- Fernández-Baca, JC y Martin AS. 2007. Indigenous Peoples and Protected Areas Management. Innovations in Conservation Series, Parks in Peril Program. The Nature Conservancy, Arlington, VA, USA, 2op.

- Giglio L, Randerson JT, van der Werf GR. 2013. Analysis of daily, monthly, and annual burned area using the fourth-generation global fire emissions database (GFED4). Journal Of Geophysical Research, 118(1):317-328.

- Holling CS. 1973. Resilience and stability of ecological systems. Annual Review of Ecology, Evolution, and Systematics, 4:1-23.
- Morton DC, Defries RS, Randerson JT, Giglio L, Schoeder W. van der Werf GR. 2008. Agricultural intensification increases deforestation fire activity in Amazonia. Global Change Biology, 14:2262-2275.

- Murcia U, Medina R, Rodriguez JM, Castellanos H, Hérnandez A, Herrera E. 2014. Monitoreo de los bosques y otras coberturas de la Amazonia Colombiana, a escala 1:100.000. Datos del periodo 2012. Instituto Amazónico de Investigaciones Científicas Sinchi, Bogotá, D. C, 190 p.

- Nelson A y Chomitz K. 2011. Effectiveness of Strict vs. Multiple Use Protected Areas in Reducing Tropical Forest Fires: A Global Analysis Using Matching Methods. Plos One, 6(8):1-14

- Nepstad DC, Schartzman S, Bambeger B, Santilli M, Ray D, Schlesinger P, Lefebvre P, Alencar A, Prinz E, Fiske G, Rolla A. 2006. Inhibition of Amazon Deforestation and Fire by Parks and Indigenous Lands. Conservation Biology, 20(1):65-73.

- Oliveira PJC, Asner GP, Knapp DE, Almeyda A, Galván-Gildemeister R, Keene S, Raybin RF, Smith RC. 2007. Land-use allocation protects the Peruvian Amazon. Science, 317:1233-1236.

- Plan Básico de Ordenamiento Territorial, municipio de San José del Guaviare. 2000. Documento técnico, San José del Guaviare, 16op.

- Plan de desarrollo del Guaviare. 2004. Ordenanza No 008 del 2004. Departamento del Guaviare, 79p.

- Pereira P, Mierauskas P, Úbeda J, Mtaix-Solera J, Cerda A. 2012. Fire in Protected Areas - the Effect of Protection and Importance of Fire Management. Environmental Research, Engineering and Management, 1(59):52-62.

- Ponce C y Curonisy Y. 2008a. La Categoría VI de la UICN en América Latina: Área Protegida para el Manejo de Recursos. Programa FAO/OAPN. Fortalecimiento del Manejo Sostenible de los Recursos Naturales en las Áreas Protegidas de América Latina54p.

- Ponce C y Curonisy. 2008b. Manual de Capacitación La Categoría VI de la UICN en América Latina: Área Protegida para el Manejo de Recursos. Programa FAO/OAPN. Fortalecimiento del Manejo Sostenible de los Recursos Naturales en las Áreas Protegidas de América Latina, 48p.

- Secretariat of the Convention on Biological Diversity. 2009. Biodiversity, Development and Poverty Alleviation: Recognizing the Role of Biodiversity for Human Well-being. CBD, Montreal, 52p.

- Solano P. 2009. Marcos Regulatorios Nacionales de Áreas Protegidas: Perú. IUCN-EPLP No. 81, 55p.

- Suarez C, Prüssmann J, Lopez C, Abud M, Guevara O, Vergara A. 2015 Vulnerability Analysis of the Amazon Biome and its Protected Areas. Amazon Vision, 15p.

- Tittensor DP, Walpole M, Hill SL, Boyce DG, Britten GL, Burgess ND, Butchart SHM, Leadley PW, Regan EC, Alkemade R, Baumung R, Bellard C, Bouwman L, Bowles-Newark NJ, Chenery AM, Cheung WWL, Christensen V, Cooper D, Crowther AR, Dixon MJR, Galli A, Gaveau V, Gregory RD, Gutierrez NL, Hirsch TL, Höft R, Januchowski-Hartley SR, Karmann $M$, Krug CB, Leverington FJ; Loh J, Lojenga RK, Malsch K, Marques A, Morgan DHW, Mumby PJ, Newbold T, Noonan-Mooney K, Pagad SN, Parks BC, Pereira HM, Robertson T, Rondini C, Santini L, Scharlemann JPW, Schindler S, Sumalia UR, Teh LSL, van Kolck J, Visconti P, Ye Y.2014. A mid-term analysis of progress toward international biodiversity targets. Science, 346:241-244.

- WCMC. 2010.World Database on Protected Areas. Disponible en www.wdpa.org. World Conservation Monitoring Centre, Cambridge UK.

- WDPA. 2015. World Database on Protected Areas. Disponible en: http://www.protectedplanet.net

- White CA, Perrakis DDB, Kafka VG, Ennis T. 2011. Burning at the edge: Integrating biophysical and Ecocultural fire process in Canada's Parks and protected areas. Fire Ecology, 7(1):74-106. 